\title{
柔性祄底/刚性薄膜应力褶皱有序结构形成的动态 过程
}

\author{
李超荣 ${ }^{(1)}$ ，徐庆 ${ }^{(1)}$ ，鲁年鹏 ${ }^{(2)}$, 董文钧 ${ }^{(1)}$ ，曹则贤 ${ }^{(2)}$ \\ (1) 浙江理工大学物理系和先进纺织材料与制备技术教育部重点实验室, 杭州 310018 ; \\ (2) 中国科学院物理研究所, 北京 100190 \\ *联系人, E-mail: crli@zstu.edu.cn
}

收稿日期: 2011-11-19; 接受日期: 2012-04-28; 网络出版日期: 2012-08-09

国家自然科学基金(批准号: 91122022,10874153, 51172209, 50972130)和浙江理工大学科研启动基金(编号: 0713037)资助项目

摘要对于柔性衬底/刚性薄膜系统, 当应力超过临界值时, 通常会产生无序皱褶. 本文在 PDMS 衬底 /Pt 薄膜系统中, 通过预制边界条件实现了皱祤图案的有序化排列, 并针对其详细过程进行了系统研究. 基于 $30^{\circ} \mathrm{C}$ 降温至 $0^{\circ} \mathrm{C}$ 的温差系统, 实时观察禇皱有序结构形成与演化动态过程. 根据力学原理分析, 研 究了褶皱起源和生长, 缺陷形成和演化规律, 以及禇皱波长和振幅随温度的变化情况. 其结果对形成大 面积高度有序的褶邹花样具有一定的指导意义.

关键词褶皱, 动态过程, 应力系统, 缺陷

PACS: $91.55 . \mathrm{Hj}$, 89.75.Kd, 46.70.-p, 68.35.Dv

doi: $10.1360 / 132011-1163$

褶皱是一种广泛存在于宏观与微观世界的自然 现象 ${ }^{[1]}$, 如起伏的山脉、失水的苹果、皮肤的皱纹等. 将此复杂的自然现象进行模型简化，可描述为在柔 性衬底上涂覆一层刚性薄膜, 当系统的温度、成分或 结构等发生变化时, 界面处会产生应力; 若应力大于 临界应力时, 系统结构失稳而形成褶皱; 若给予一定 的边界条件限制, 褶皱图案会呈有序化排列. 虽然一 些皱褶行为会对产品的性能或外观造成不良影响 ${ }^{[2]}$, 但并非所有的皱褶行为都是消极的. 如今, 人们对褶 皱现象有了更为深入的了解, 已将其广泛应用于微 流器件 ${ }^{[3]}$ 、传感器 ${ }^{[4]}$ 、光学器件 ${ }^{[5]}$ 和现代度量学 ${ }^{[6]}$ 等领
域，如可用于制造可调谐衍射光栅及测量薄膜的杨 氏模量或厚度等.

1998 年, Bowdon 等人 ${ }^{[7]}$ 在聚二甲基硅氧烷(PDMS) 祄底上沉积了一层 $\mathrm{Au}$ 薄膜, 利用两者较大的热膨胀 系数差制备了褶皱，并预制边界条件获得了有序褶 皱花样. 之后, 人们尝试了各种材料和不同途径, 如 机械拉伸释放法 ${ }^{[8]}$ 、等离子体/紫外照射法 ${ }^{[9]}$ 、热处理 法 ${ }^{[10]}$ 、溶胶凝胶干燥法 ${ }^{[11]}$ 和溶剂扩散法 ${ }^{[12]}$ 等制备了 复杂花样的褶皱 ${ }^{[13]}$ 和多级型褶皱 ${ }^{[14]} .2005$ 年, $\mathrm{Li}$ 等 人 ${ }^{[15-17]}$ 在 $\mathrm{Ag} / \mathrm{SiO}_{2}$ 核壳热应力系统中获得了三角铺 排及 Fibonacci 数螺旋花样. 2007 年, Huang 等人 ${ }^{[18]}$ 研

引用格式: 李超荣, 徐庆, 鲁年鹏, 等. 柔性补底/刚性薄膜应力褶皱有序结构形成的动态过程. 中国科学: 物理学 力学 天文学, 2012, 42: 934-940 Li C R, Xu Q, Lu N P, et al. Dynamic formation of stressed ordered wrinkle on compliant substrate/stiff film (in Chinese). Sci Sin-Phys Mech Astron, 2012, 42: 934-940, doi: 10.1360/132011-1163 
究了在毛细力作用下负载水滴的聚合物薄膜的褶皱 释放过程, 发现轮辐状褶皱长度随时间迁移逐渐减 小直至消失. 2009 年, Chung 等人 ${ }^{[19]}$ 将聚苯乙烯(PS) 祄底/改性 PS 薄膜系统进行甲苯蒸汽附着处理, 观察 到轮辐状和箭靶状褶皱花样首先在缺陷位点处成核, 之后呈辐射状扩散. 2011 年, Duan 等人 ${ }^{[20]}$ 计算模拟了 单层石墨烯的褶皱花样随剪切应力施加的变化过程, 其从自由边缘区域产生后向中心区域传播. 简而言 之, 当前大量研究工作主要集中于系统结构失稳前 后两种稳定状态的研究, 而较少涉及系统如何进行 稳态的动态转变.

本文利用温度控制热应力的方法, 实时研究了 PDMS 柔性祄底/Pt 刚性薄膜系统的皱褶形成与演化 的动态过程, 并研究了不同降温过程对褶皱形貌的 影响. 通过对模型体系规律性的研究, 可以解释有序 褶皱中缺陷形成原理, 为制备大面积有序结构提供 了可能性.

\section{1 实验方法}

\section{1 预制边界条件限制的 PDMS 衬底/Pt 薄膜系统 的制备}

将 PDMS(Dow Corning, Sylgard ${ }^{\circledR}$ 184)预聚体和 固化剂以质量比 10:1 的比例混合并搅拌均匀, 混合 物浇注正性光刻胶模板(如图 1(a), URE-2000/25 型紫 外光刻机光刻), 如图 1(b)放置. 整体在室温真空(真 空度 $0.09 \mathrm{MPa}$ )下脱气泡约 $30 \mathrm{~min}$, 然后在 $70^{\circ} \mathrm{C}$ 常压 下固化约 $4 \mathrm{~h}$. 待其降至室温后, 将固化的 PDMS 从 光刻胶模板上剥离, 得到与光刻胶模板图案互补的 预制图案 PDMS 样品, 如图 1(c)所示. 将 $30^{\circ} \mathrm{C}$ 的 PDMS 样品放置于离子溅射仪(HITACHI E-1010)下 贼射一层约 $6 \mathrm{~nm}$ 厚的薄膜, 得到有边界条件限制的 PDMS 衬底/Pt 薄膜的双层结构样品, 如图 1(d).

\section{2 褶皱的动态过程}

如图 1(e)所示为实验室自制装置示意图, PDMS 祄底/Pt 薄膜样品紧贴着 $2 \mathrm{~mm}$ 厚的透明玻璃放置, 箭 头表示出入水口. 关闭闸门II并打开闸门I, 向腔体内 持续注入 $0^{\circ} \mathrm{C}$ 冰水混合液. 利用光学显微镜(Olympus $\mathrm{BX} 51)$ 对样品进行实时观察, 从水面与玻璃相接触时 刻开始以 $3 \mathrm{~s} /$ 帧的速度拍摄样品形貌.

\section{3 不同降温速率的褶皱制备}

为研究降温速率对褶皱花样的影响, 本文设计 了骤冷和缓慢冷却两组定性对比实验. 先将 $\mathrm{A}$ 和 $\mathrm{B}$ 两组具有相同图案的 PDMS 祄底/Pt 薄膜样品进行 $30^{\circ} \mathrm{C}$ 水浴恒温预处理, 以消除残余应力. 然后如图 1(f)所示, 打开闸门I和II, 不断注入冰水混合物并使 之淹没玻璃上表面, 接着将 $\mathrm{A}$ 组样品直接放置于冰 水覆盖的玻璃表面使之达到瞬间冷却效果. 而 $\mathrm{B}$ 组 样品如图 1(g)所示, 先打开闸门I和II, 向腔体内注入 $30^{\circ} \mathrm{C}$ 水直至淹没玻璃上表面, 接着将 $\mathrm{B}$ 组样品直接 放置于此水域覆盖的玻璃表面, 缓慢注入 $0^{\circ} \mathrm{C}$ 冰水, 实时监测出水口水温是否达到 $0^{\circ} \mathrm{C}$, 冷却过程控制在 $30 \min$ 左右.

\section{2 实验结果与讨论}

\section{1 褶皱的形成条件}

临界应力是描述褶皱现象的重要参量之一. 对 于热应力作用的柔性祄底/刚性薄膜系统, 初始状态 为光滑的平面结构, 随着温度的逐渐降低, 在衬底与 薄膜的界面处热应力积聚增大直至超过临界应力, 原平衡状态破坏而产生褶皱。一般情况下, 其褶皱图

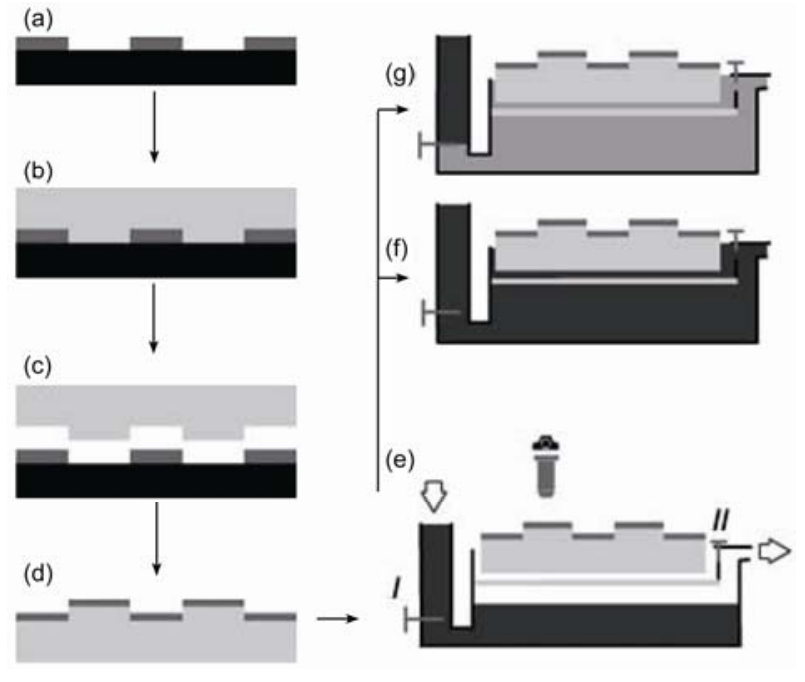

图 1 实验流程示意图

（a) 正性光刻胶掩模板; (b) 浇注; (c) 剥离; (d) 溅射; (e) 冷却; (f) A 组样品放置(骤冷); ( g) B 组样品放置(缓慢冷却)

Figure 1 The scheme of experimental procedure: (a) positive photoresist mask; (b) casting; (c) peeling; (d) sputtering; (e) cooling; (f) setting of sample group A (fast cooling); (g) setting of sample group B (slow cooling). 
案为无序花样, 当存在直线边界条件时, 褶皱图案会 呈一维条状有序结构排列. 其形成原理图如图 2(a)和 (b)所示, $x$ 轴的边界长度大于 $y$ 轴, $\lambda$ 和 $A$ 是描述褶皱 现象的两个特征参量 ${ }^{[21]}$, 分别表示褶皱波长和振幅.

由理论力学分析可知, 系统失稳的临界应力为

$$
\sigma_{\text {crit }}=\left(\frac{9 E_{\mathrm{f}} E_{\mathrm{s}}^{2}}{64\left(1-v_{\mathrm{f}}^{2}\right)\left(1-v_{\mathrm{s}}^{2}\right)^{2}}\right)^{\frac{1}{3}},
$$

其中 $E$ 为杨氏模量 $\left(E_{\mathrm{f}}>E_{\mathrm{s}}\right), v$ 为泊松比, 右下角标的 $\mathrm{f}$ 表示薄膜(film), $\mathrm{s}$ 表示祄底(substrate). 在 PDMS 祄底 $/ \mathrm{Pt}$ 薄膜系统中, $E_{\mathrm{f}}=169 \mathrm{GPa}, v_{\mathrm{f}}=0.38, E_{\mathrm{s}}=20 \mathrm{MPa}, v_{\mathrm{s}}$ $=0.48$. 可得, 临界应力 $\sigma_{\text {crit }} \approx 2.66 \times 10^{8} \mathrm{~Pa}$.

PDMS 祄底 $/ \mathrm{Pt}$ 薄膜系统由初始温度 $30^{\circ} \mathrm{C}$ 向 $0^{\circ} \mathrm{C}$ 降温, 系统稳定后祄底与薄膜的界面处产生的热应 力为

$$
\sigma_{0}=\frac{E_{\mathrm{f}}\left(\alpha_{\mathrm{s}}-\alpha_{\mathrm{f}}\right)\left(T_{\mathrm{d}}-T_{\mathrm{u}}\right)}{1-v_{\mathrm{f}}},
$$

其中 $T_{\mathrm{d}}$ 为初始溅射温度 $303 \mathrm{~K}, T_{\mathrm{u}}$ 为最终温度 $273 \mathrm{~K}$; $\alpha$ 为热膨胀系数, $\alpha_{\mathrm{f}}=9.00 \times 10^{-6} \mathrm{~K}^{-1}, \alpha_{\mathrm{s}}=3.25 \times 10^{-4} \mathrm{~K}^{-1}$. 可得, 积聚的热应力 $\sigma_{0} \approx 2.58 \times 10^{9} \mathrm{~Pa}$, 其远大于临界 应力 $\sigma_{\text {crit }}$, 此时褶皱已经产生. 此外要考虑镀膜过程 中, PDMS 受到高温的 Pt 颗粒作用, 祄底表面发生改 性产生 $E_{\mathrm{s}}$ 较大的缓冲层, 使得临界应力增大. 只有在 $\sigma_{0} \gg \sigma_{\text {crit }}$ 情况下, 可比较容易地通过光学显微镜观察 到褶皱花样.

\section{2 褶皱形成的动态过程}

为更好地研究应力褶皱有序结构的形成过程, 我们实时观察并拍摄了边界条件限制作用下禇皱条 纹形成的动态过程, 并将实验结果图进行了快速傅 里叶变换(FFT), 主要目的是将周期性连续的时域信

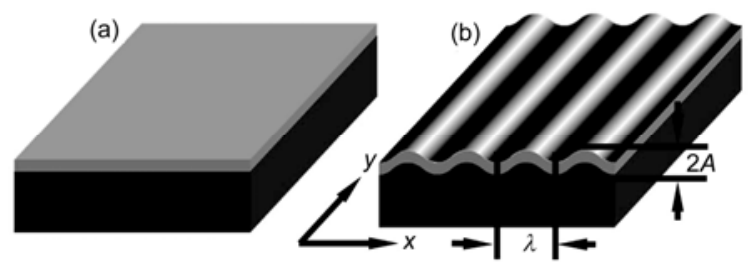

图 2 (a) 柔性衬底/刚性薄膜系统的初始状态图; (b) 最 终状态图

Figure 2 The stressed system of compliant substrate/stiff film. (a) The initial state; (b) the final state.
号转换成直观的离散频域信号, 结果如图 3 所示. 记 冰水浴与玻璃片恰未接触的时刻为动态过程的初始 时刻，此时 PDMS 祄底/Pt 薄膜系统正处于 $30^{\circ} \mathrm{C}$ 环境 中. 视野中主要有 4 块长条形小区域, 以 $y$ 轴方向上 的第三个独立小区域为观察对象.

在 $3 \mathrm{~s}$ ，水面接触玻璃面，系统开始降温，观察区 表面大部分区域呈平整光滑状态, 但存在少量明显 的凹坑缺陷，如图 3 右下角放大图.

在 $54 \mathrm{~s}$, 观察区表面粗䊚化, 出现了一些 $y$ 轴走 向的一维条状褶皱, 主要集中分布于靠近凹坑和直 线边界区, 约在距离直线边界 1-6 $\mu \mathrm{m}$ 范围内. 褶皱 的伸展可通过明暗分布观察, 靠近凹坑与直线边界 区域线条伸长，而远离边界区域表面平滑. 褶皱互不 干扰地从上下直线边界区向中间区伸展, 或从凹坑 分别向上下边界区伸展, 如图 3 右下角放大图所示. 此时, 褶皱生长方向相同而生长速率各异.

在 $75 \mathrm{~s}$, 观察区内靠近直线边界区的褶皱条纹沿 $y$ 轴方向进一步向中心伸展并加深, 靠近凹坑区的褶 皱向边界区域伸展并停止于边界处, 如图 3 右下角放 大图所示. 对上下边界处的褶皱条纹分别进行延伸 处理, 发现部分条纹并不相重合, 存在错位现象. 由 FFT 图可知, 褶皱在 $x$ 轴方向上已经有规律化排列的 趋势, 但并不明显. 此时, 褶皱振幅加强, 而褶皱初 始生长点互不干扰.

在 $111 \mathrm{~s}$, 观察区所有区域均被 $y$ 轴走向的褶皱 所覆盖, 从上直线边界区伸展出的褶皱条纹与下直 线边界区伸展处的错位褶皱条纹联接, 成为一条弯 曲的褶皱条纹. 测量得平均波长为 $5.8 \mu \mathrm{m}$. 由 FFT 图 可知, 褶皱在 $x$ 轴方向上排列规律性加强.

在 $129 \mathrm{~s}$, 观察区所有褶皱明暗对比进一步加强, 其中箭头所指示是褶皱缺陷，其破坏了褶皱排列的 规律性, 所在位置与凹坑位置无关. 靠近缺陷处的弯 曲条纹弧度减小而趋近于直线条纹, 走向偏离边界 $90^{\circ}$ 方向, 远离缺陷处的褶皱条纹与边界互相垂直.

在 $150 \mathrm{~s}$, 观察区的褶皱在 $y$ 轴方向上的长度与 明暗对比度与前一时刻相比较已无明显变化, 平均 波长为 $5.8 \mu \mathrm{m}$, FFT 图也无明显变化, 说明此 PDMS 祄底 $/ \mathrm{Pt}$ 薄膜系统已基本趋于稳定.

可将褶皱现象理解为无脱层的膜趐曲行为, 褶 皱的形貌变化主要取决于 $y$ 轴方向的弯矩 ${ }^{[22]}$. 弯矩经 修正后可表示为 

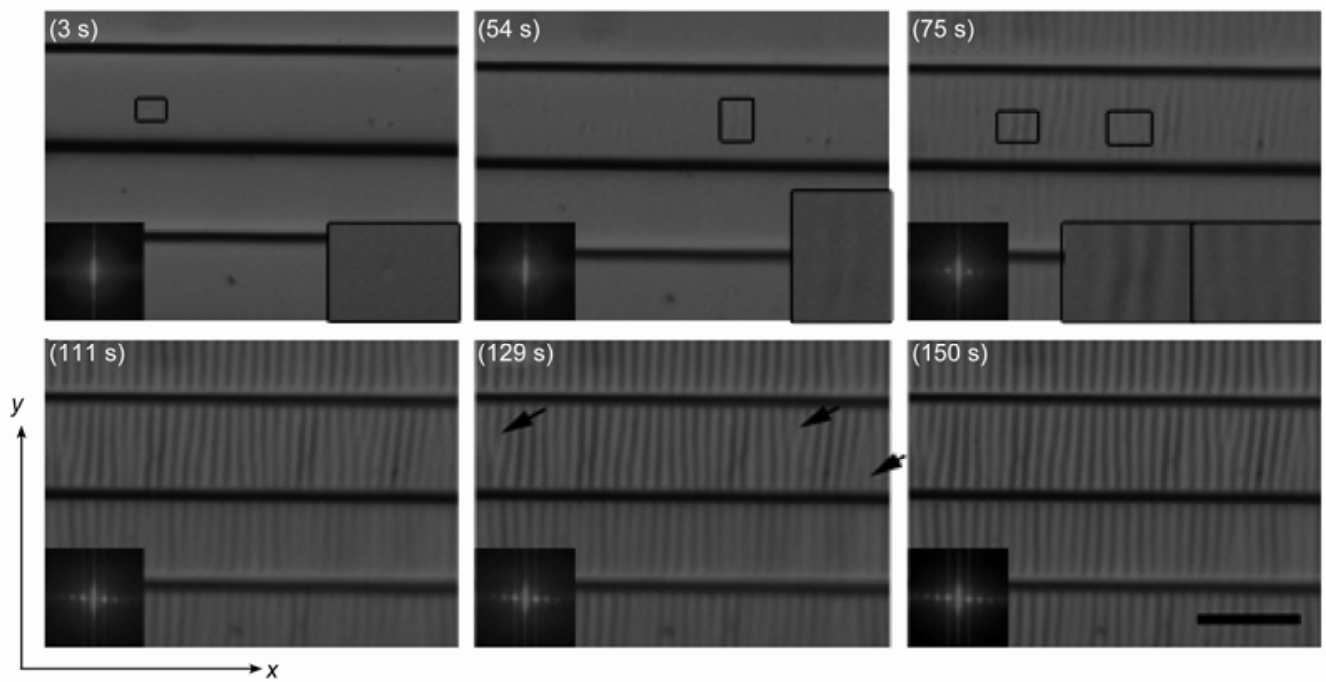

图 3 PDMS 衬底/Pt 薄膜系统第 3, 54, 75, 111, 129, $150 \mathrm{~s}$ 时的褶皱演化过程状态图

左下角为快速傅里叶变换图, 右下角为黑色框内的放大图, 标尺为 $40 \mu \mathrm{m}$

Figure 3 The evolution of buckling patterns of the PDMS/Pt stressed system at 3, 54, 75, 111, 129 and $150 \mathrm{~s}$. The inset at the down-left corner is the Furrier transformed pattern and the down-right is the enlarged image, scale bar $40 \mu \mathrm{m}$.

$$
m_{y}(y)=\frac{\sigma_{0 y} h}{\sqrt{3}} \frac{(\lambda / 2)^{2}}{(y+\lambda / 2)^{2}} \sqrt{\frac{(y+\lambda / 2)^{2}}{(\lambda / 2)^{2}}-1},
$$

其中 $\sigma_{0 y}$ 为 $y$ 轴方向的热应力, $y$ 为 $y$ 轴方向上距离边 界的距离. 可知, 若应力 $\sigma_{0 y}$ 一定时, 在边界处 $y=0$, 弯矩 $m_{y}(0)=0$, 随后 $m_{y}$ 随 $y$ 值增大而增大; 在离边界 $(\sqrt{2}-1) \lambda / 2$ 位置, $m_{y}$ 达到最大值, 此处翅曲现象最 明显, 后 $m_{y}$ 随 $y$ 值增大而减小最后渐趋于 0 . 由此可 知, 靠近边界区域的应力集中, 首先观察到屈曲行为. 当应力随温差增大而逐渐增加, 弯矩 $m_{y}$ 相应增大, 在远离边界区域也可以观察到褶皱现象.

在观察区域被褶皱覆盖后, 褶皱条纹明暗对比 增大，其与褶皱振幅有关. 振幅公式为 ${ }^{[21]}$

$$
A=h\left[\frac{\varepsilon_{0}}{\varepsilon_{\mathrm{c}}}-1\right]^{\frac{1}{2}}=h\left[\frac{\sigma_{0} / E_{\mathrm{f}}}{\sigma_{\mathrm{c}} / E_{\mathrm{f}}}-1\right]^{\frac{1}{2}},
$$

其中薄膜厚度 $h$ 为 $6 \mathrm{~nm}, \varepsilon_{0}$ 表示热应变, $\varepsilon_{\mathrm{c}}$ 表示临界应 变. 由此可知, 振幅只和热应力与临界应力比值有关, 即材料属性不变时只与温差有关. 当温差 $\Delta T$ 值很小 时, 例如 $\Delta T=T_{\mathrm{u}}-T_{\mathrm{i}}=1 \mathrm{~K}, \sigma_{0}<\sigma_{\text {crit }}$, 此时无褶皱现象; 当 $\Delta T=4 \mathrm{~K}$, 此时 $\sigma_{0}>\sigma_{\text {crit }}$, 褶皱已经产生, 但 $A=3.25$ $\mathrm{nm}$, 振幅极小, 此时褶皱不易通过光学显微镜观察. 若 $\Delta T=30 \mathrm{~K}, A=17.70 \mathrm{~nm}$, 此时褶皱现象已经非常 明显.
在观察区域被褶皱覆盖后，褶皱条纹波长无明 显变化. 褶皱波长公式为 ${ }^{[21]}$

$$
\lambda=2 \pi h\left[\frac{\left(1-v_{\mathrm{s}}^{2}\right) E_{\mathrm{f}}}{3\left(1-v_{\mathrm{f}}^{2}\right) E_{\mathrm{s}}}\right]^{\frac{1}{3}},
$$

可知, 褶皱理论波长只与材料属性和薄膜厚度有关, 与温度变化无关. 褶皱在覆盖视野区后褶皱条纹数 目无变化, 与理论相符.

褶皱行为严格意义上是一个热力学过程, 内能 $U$ 应正比于形变体积 $V$. 当相向两褶皱条纹刚联接为 一条弯曲褶皱条纹时, 褶皱条纹长度 $L$ 较大使得形变 体积 $V$ 较大, 内能较高, PDMS 祄底/Pt 薄膜系统结构 不稳定，而向更稳定结构演变. 当褶皱条纹呈直线时, $L$ 最小, 褶皱结构最稳定.

由此可见，在热应力作用下具有边界条件限制 的柔性祄底/刚性薄膜系统褶皱产生与演化规律为: 褶皱首先在距离边界, 包括人为边界和工艺缺陷的 $(\sqrt{2}-1) / 2$ 个波长处生成褶皱，然后褶皱以不同的速 率从各自靠近边界区域独立地向非边界区域生长且 生长方向垂直于边界, 其中相向生长的毗邻褶皱条 纹排列严格遵循波长公式和能量最低原则, 两者前 端联接成一条弯曲褶皱条纹后又演化成直线条纹, 同时伴随着少量缺陷的产生，随着应力的增加，褶皱 
的振幅增大而波长不变.

褶皱的产生与演化规律为褶皱缺陷的形成与演 化提供了依据. 由于从两边界附近区域初始形成褶 皱条纹是独立的，一些褶皱条纹初始生长点会存在 剧烈错位, 且各处褶皱条纹拥有不同生长速率, 如图 4(a)所示. 受应力持续作用，上下边界处褶皱继续生 长, 下边界褶皱会与上边界相邻褶皱中生长速率快 者联接在一起成为一条弯曲褶皱. 若恰上边界两侧 褶皱的生长速率较快, 而中间生长速率较慢, 则速率 慢的褶皱会成为缺陷, 如图 4(b). 根据能量最低原则, 两侧曲率大的褶皱曲线会演化为曲率较小的褶皱条 纹, 最终形成如图 4(c).

\section{3 降温速度对褶皱缺陷的影响}

缺陷率是指单位面积的缺陷个数, 是评价褶皱 周期性效果和有序程度的重要标准. 如图 5(a), A 组 样品降温过程可认为瞬间降温过程, 其缺陷率为 78.34 个 $/ \mathrm{mm}^{2}$, 褶䏢波长为 $(6.5 \pm 0.5) \mu \mathrm{m}$. 如图 5(b),

(a)

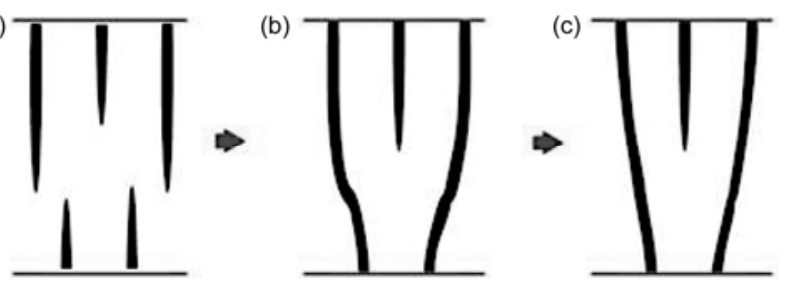

图 4 缺陷形成与演化示意图

(a)襀皱条纹的互错生长; (b) 条纹的联接; (c) 条纹的演化, 细线表 示边界, 粗线表示褟䏢的凸起

Figure 4 The schematic diagram of the formation and evolution of wrinkles: (a) malposed growth, (b)connection, (c) evolution, the thin lines indicate boundaries, the thick lines indicate the bulges of wrinkles.
B 组样品为缓慢降温过程, 其缺陷率为 33.57 个 $/ \mathrm{mm}^{2}$, 褶皱波长为 $(6.5 \pm 0.3) \mu \mathrm{m}$. 可知, 系统降温速度越快, 应变的释放速度越快, 缺陷率越高, 褶皱的周期性效 果越差, 有序化程度越低. 由褶皱缺陷的生长机理可 知, 在快速应变释放过程中, 褶皱生长可到达的最快 速度增大, 此时褶皱生长速度差增大, 形成缺陷的几 率增加; 反之, 在较慢应变释放情况下, 褶皱所能达 到的最大生长速度比较小, 生长速度易趋于一致, 不 易形成缺陷.

实质上，缺陷率取决于应变的释放速度，理论上 若释放速度无限慢可得到具有完美周期的褶皱图案. 那么减小降温速率可得到低缺陷率褶皱，对形成大 面积的有序化程度高的褶皱花样有非常积极的意义.

\section{3 结论}

本文在预制边界条件的 PDMS 柔性祄底上溅射 一层均匀 $\mathrm{Pt}$ 刚性薄膜, 由 $30^{\circ} \mathrm{C}$ 初始温度降温至 $0^{\circ} \mathrm{C}$, 获得了一维有序褶皱条纹, 并实时观察了有序褶皱 条纹形成和演化的动态过程. 实验结果表明: PDMS 祄底/Pt 薄膜系统初始状态为平滑结构, 随温度的降 低, 首先在离边界 $(\sqrt{2}-1) / 2$ 个波长处或凹坑区域出 现褶皱. 然后各边界区域附近的褶皱以不同的速率 独立地向中心生长且方向垂直于边界, 相向快速生 长的两褶皱条纹联接成一条曲线褶皱条纹. 根据能 量最低原则, 演化为结构更为稳定的直线褶皱条纹, 其中, 慢速生长的单条纹成为了缺陷. 随着时间的迁 移，褶皱呈周期性排列，褶皱条纹振幅增大而波长不 变, 最终达到稳定状态. 不同降温过程对褶皱条纹有一 定的影响, 降温速率越小则缺陷率越低, 有序度越好.
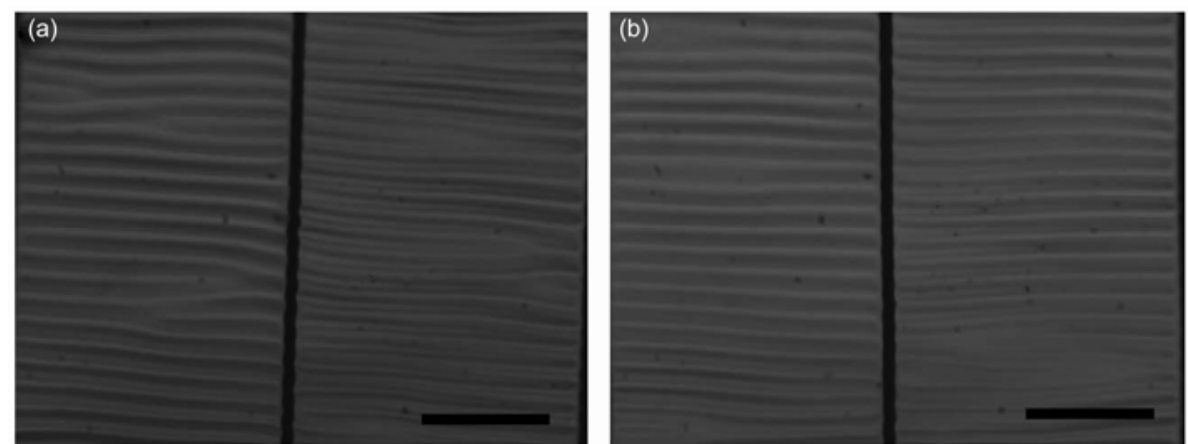

图 5 A 组样品褶皱形貌(a)和 B 组样品的褶皱图(b)

标尺为 $40 \mu \mathrm{m}$

Figure 5 The buckling morphology. (a) Sample group A, (b) sample group B, scale bar $40 \mu \mathrm{m}$. 


\section{参考文献}

1 Miller A F. Exploiting wrinkle formation. Science, 2007, 317: 605-606

2 Liang W, Yang W M, Cheng X F, et al. The effect of $\mathrm{Gd}_{2} \mathrm{Ba}_{4} \mathrm{CuNbO}_{\mathrm{y}}$ doping on the properties of single domain GdBCO bulk superconductors (in Chinese). Sci Sin-Phys Mech Astron, 2011, 41: 201-206 [梁伟, 杨万民, 程晓芳, 等. $\mathrm{Gd}_{2} \mathrm{Ba}_{4} \mathrm{CuNbO}_{y}$ 掺杂对单畴 GdBCO 超导块材性能的影响. 中国科学: 物理学 力学 天文学, 2011,41(2): 201-206]

3 Chung J Y , Chastek T Q, Fasolka M J, et al. Quantifying residual stress in nanoscale thin polymer films via surface wrinkling. ACS Nano, 3(4): 844-852

4 Liedtke A, Lei C H, Neill O M, et al. One-step photoembossing for submicrometer surface relief structures in liquid crystal semiconductors. ACS Nano, 2010, 4(6): 3248-3253

5 Chung J Y, Nolte A J, Stafford C M. Surface wrinkling: A versatile platform for measuring thin-film properties. Adv Mater, 2011, 23(3): 349-368

6 Wilder E A, Guo S, Guo S L, et al. Measuring the modulus of soft polymer networks via a buckling-based metrology. Macromolecules, 2006, 39: 4138-4143

7 Bowden N, Brittain S, Evans A G, et al. Spontaneous formation of ordered structures in thin films of metals supported on an elastomeric polymer. Nature, 1998, 393: 146-149

8 Khare K, Zhou J H, Yang S. Tunable open-channel microfluidics on soft poly(dimethylsiloxane) (PDMS) substrates with sinusoidal grooves. Langmuir, 2009, 25(21): 12794-12799

9 Takahashi M, Uemura K, Maeda T, et al. Bottom-up and top-down approach for periodic microstructures on thin oxide films by controlled photo-activated chemical processes. J Sol Gel Sci Technol, 2008, 48: 182-186

10 Wilhelm T H, Ned B, Patrick O, et al. Ordering of spontaneously formed buckles on planar surfaces. Langmuir, 2000, 16: 3497-3501

11 Garnier M G, Croll A B, Davis C S, et al. Contact-line mechanics for pattern control. Soft Matter, 2010, 6: 5789-5794

12 Chah S, Noolandi J, Zare R N. Undulatory delamination of thin polymer films on gold surfaces. J Phys Chem B, 2005, 109(41): 19416-19421

13 Hendricks T R, Wang W, Lee I. Buckling in nanomechanical films. Soft Matter, 2010, 6: 3701-3706

14 Efimenko K, Rackaitis M, Manias E, et al. Nested self-similar wrinkling patterns in skins. Nat Mater, 2005, 4: 293-297

15 Li C R, Zhang X N, Cao Z X. Triangular and Fibonacci number patterns driven by stress on core/shell microstructures. Science, 2005, 309: 909-911

16 Li C R, Ji A L, Cao Z X. Stressed Fibonacci spiral patterns of definite chirality. Appl Phys Lett, 90, 2007: 164102

17 Li C R, Ji A L, Gao L, et al. Stressed triangular tessellations and Fibonacci parastichous spirals on $\mathrm{Ag}_{\text {core/SiO}}$ shell microstructures. Adv Mater, 2009, 21: 1-6

18 Huang H S, Juszkiewicz M, Jeu H W, et al. Caplillary wrinkling of floating thin polymer films. Science, 2007, 317: 650-653

19 Chung J Y, Nolte A J, Stafford C M. Diffusion-controlled, self-organized growth of symmetric wrinkling patterns. Adv Mater, 2009, 21: 1358-1362

20 Duan W H, Gong K, Wang Q. Controlling the formation of wrinkles in a single layer graphene sheet subjected to in-plane shear. Carbon, 2011, 49: 3107-3112

21 Schweikart A, Fery A. Controlled wrinkling as a novel method for the fabrication of patterned surfaces. Microchim Acta, 2009, 165(3-4): 249-263

22 Freund L B, Suresh S. Thin Film Materials: Stress, Defect Formation and Surface Evolution. Cambridge: Cambridge University Press, 2003. $382-385$ 


\title{
Dynamic formation of stressed ordered wrinkle on compliant substrate/stiff film
}

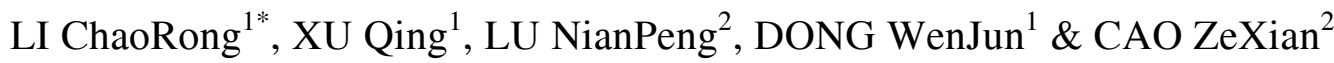 \\ ${ }^{1}$ Department of Physics and Key Laboratory of Advanced Textile and Manufacturing Technology, Zhejiang Sci-Tech University, \\ Hangzhou 310018, China; \\ ${ }^{2}$ National Laboratory for Condensed Matters, Institute of Physics, Chinese Academy of Sciences, Beijing 100190, China
}

\begin{abstract}
A compliant substrate/stiff film stressed system usually forms disordered wrinkle when the stress exceeds critical value. In this paper, the ordered one-dimensional wrinkle patterns were realized by the prefabricated boundaries in the PDMS substrate/Pt film system. Meanwhile, the detailed formation processes were also systematically investigated. By cooling the system from $30^{\circ} \mathrm{C}$ down to $0^{\circ} \mathrm{C}$, we have in situ observed the dynamic formation and evolution process of the ordered wrinkle structure. According to the mechanics principles, some processes including the initiation and development of the wrinkle, the formation and evolution of the defect as well as the variation of wavelength and amplitude with temperature were studied. The results offer some clues to the formation of large-scaled high ordered wrinkle patterns.
\end{abstract}

wrinkle, dynamic process, wavelength, defect

PACS: $91.55 . \mathrm{Hj}$, 89.75.Kd, 46.70.-p, 68.35.Dv

doi: $10.1360 / 132011-1163$ 